\title{
Argumentos Intuitivos De Futuros Profesores: Una Experiencia Con Probabilidad
}

\section{Intuitive Arguments of prospective teachers: An Experience With Probability}

\author{
J. Marcos López-Mojica* \\ UAGro \\ Lilia P. Aké** \\ UAQ
}

\begin{abstract}
Resumen
Desde una perspectiva epistemológica y cognitiva, en el presente informe de investigación se analizan los argumentos intuitivos producidos por futuros profesores de matemáticas del nivel bachillerato en una experiencia con un fenómeno aleatorio de manera sistemática. Los elementos teóricos se enmarcan en el desarrollo conceptual de la probabilidad (intuitivo, clásico, frecuencial y axiomático), la intuición, esquema de prueba empírico y la argumentación. Con un enfoque cualitativo, se desarrolló una actividad de enseñanza sobre la mezcla aleatoria con 12 participantes de una universidad pública mexicana. El método de la experienciación permitió la instrumentación de guiones de clase, guión de la actividad de referencia, hojas de control y videograbación. Los resultados sugieren una configuración de argumentos intuitivos respecto a la medida de probabilidad, espacio muestra, combinatoria y ley de los grandes números, que permitieron evolucionar a nociones matemáticas relativas a la probabilidad.
\end{abstract}

Palabras clave: Significados de probabilidad, Argumentos intuitivos, Futuros profesores.

\begin{abstract}
From an epistemological and cognitive perspective, this research report analyzes the intuitive arguments produced by future mathematics teachers at the baccalaureate level in an experience with a random phenomenon in a systematic way. The theoretical elements are framed in the conceptual development of probability (intuitive, classical, frequency and axiomatic), intuition, empirical test scheme and argumentation. With a qualitative approach, a teaching activity on random mixing was developed with 12 participants from a Mexican public university. The method of experience allowed the instrumentation of class scripts, script of reference activity, control sheets and video recording. The results suggest a configuration of intuitive arguments regarding the measure of probability, sample space, combinatorial and law of large numbers, which allowed us to evolve to mathematical notions related to probability.
\end{abstract}

Keywords: Meanings of probability, Intuitive arguments, Prospective teachers.

\footnotetext{
* Doctor en Ciencias especialidad Matemática Educativa por el Centro de Investigación y de Estudios Avanzados del IPN (Cinvestav-IPN). Profesor-Investigador de la Universidad Autónoma de Guerrero (UAGro), Guerrero, México. E-mail: mojicajm@gmail.com.

** Doctora en Didáctica de la Matemática por la Universidad de Granada (UGR), España. Posdoctorante en la Universidad Autónoma de Querétaro (UAQ), Querétaro, México. E-mail: lake86@gmail.com.
} 


\section{Introducción}

El gran avance tecnológico y científico que vive actualmente la sociedad ha generado el acceso a la información sin restricciones. Esto debido al desarrollo de la teoría de la probabilidad (Narens, 2007). Sus repercusiones competen en la ciencia, la filosofía, en la actividad humana, incluso en tendencias en el arte (Ojeda, 2003). Por ejemplo en psicología, la probabilidad y la estadística han jugado un papel importante no sólo para otorgarle el status de disciplina, sino en la investigación de procesos del pensamiento del individuo (Fischbein, 1975).

Lo anterior exige que los ciudadanos deban estar preparados para el tratamiento de la información. Los sistemas complejos y masivos demandan a los habitantes una formación en estocásticos ${ }^{1}$ para la toma de decisiones. El reconocimiento de tal situación obliga al sistema educativo nacional introducir temas de probabilidad y de estadística en toda la educación básica.

Los cambios curriculares que han sufrido varios países han considerado la problemática de agregar elementos de probabilidad y de estadística como contenido disciplinar (Vásquez y Alsina, 2017). Esto se ha reflejado también en la formación de profesores, por ejemplo en el contexto mexicano Martínez y Ojeda (2017) señalan una reciente incorporación de estos temas en el currículo para profesores de primaria regular en la materia Procesamiento de la Información Estadística. Sin embargo, aún prevalece la incomprensión de estocásticos tanto en los estudiantes como en los futuros profesores (López-Mojica, Ojeda y Salcedo, 2018), lo que propicia sesgos del pensamiento (Kahneman, Slovic y Tversky, 1982; Ojeda, 1994).

La necesidad del estudio de la probabilidad y de la estadística es relevante, por un lado, desarrollar un pensamiento probabilístico impone un modo distinto de pensar (Fischbein, 1975), porque el pensamiento se abre a lo posible (Piaget, 1982) y en consecuencia se desarrolla un pensamiento crítico. Además, los temas de estocásticos convocan la promoción de otros conceptos matemáticos (Steinbring, 2005) y permiten dotarle de otro sentido por su uso, por lo que se establece una red conceptual para la constitución de un pensamiento matemático que considera la intervención del azar (Heitele, 1975; Steinbring, 1991).

A más de 30 años Steinbring (1989) señaló que la probabilidad y la estadística no pueden enseñarse de la misma manera que las matemáticas escolares convencionales, pues el carácter aleatorio y la naturaleza específica de las disciplinas se pierde (Steinbring, 1989). Esta idea persiste en estudiantes, pues las miran como una colección de reglas y recetas sin explicaciones

\footnotetext{
${ }^{1}$ En el presente documento, la palabra estocásticos refiere a conceptos relativos a la probabilidad y a la estadística.
} 
o argumentos (Holmes, 1980). Por lo que es necesario comenzar a identificar la estrecha relación y complementariedad de ambas disciplinas (Batanero y Borovcnik, 2016). Conviene entonces, introducir los temas en cuestión "en un contexto más amplio de situaciones significativas que ofrezcan oportunidades para argumentos, interpretaciones y decisiones estadísticas" (Steinbring, 1989, p. 151).

Es necesario analizar ese tipo de argumentos, interpretaciones y decisiones estocásticas que desencadenen un razonamiento probabilístico, pues identificarlas "podría permitir auxiliar a la formulación de estrategias de intervención didáctica y de herramientas de evaluación formativa orientadas a favorecer el aprendizaje de formas de validación basadas en el razonamiento deductivo" (Larios, Arellano y González, 2018, p. 281).

Tratar los temas de probabilidad con los futuros docentes del nivel medio superior, permitiría su incorporación en la formación matemática de los jóvenes y así ofrecerles un acceso al estudio de fenómenos aleatorios de manera sistemática. Es decir, se estaría preparando a los alumnos a enfrentarse a situaciones deterministas como indeterministas y garantizar, de cierta manera, una educación matemática básica integral (Ojeda, 2003).

Vásquez y Alsina (2017) expresan que un lenguaje cotidiano es pieza clave para el desarrollo de un lenguaje probabilístico y éste es un soporte para la alfabetización probabilística. Además, sugieren que los docentes empleen el uso de ideas intuitivas de los alumnos para incorporar de manera gradual los significados de la probabilidad. Lo que concuerda con lo planteado por Heitele (1975), quien recomienda presentar actividades de probabilidad y estadística lo más temprano posible para generar en el individuo intuiciones auxiliares.

El presente informe de investigación tiene como fin caracterizar los argumentos intuitivos que exhiben los futuros profesores de bachillerato cuando se les presenta una situación aleatoria. Dada la influencia de la matemática escolar determinista, es necesario romper con la tendencia de ofrecer una sola respuesta a problemas que plantean una variedad de resultados. Pues conjeturamos que una educación determinista en probabilidad provocaría en los futuros profesores argumentos con un enfoque determinista también.

\section{Elementos Teóricos De La Investigación}

En la correspondencia entre Fermat y Pascal, a más de 300 años transcurridos, se identificó una primera aproximación al estudio del azar. En ella se puede señalar que ante la 
necesidad de analizar los juegos de azar, los autores enumeraron todas las posibilidades de ocurrencia de cierto suceso, a manera de encontrar un modelo matemático que permitiera su explicación (Coutinho, 2007).

Sin embargo, autores como Hacking (1975) expresan que el concepto de probabilidad no surgió del todo de los juegos de azar. Dada su naturaleza, la idea de probabilidad implica un significado dual, "uno estadístico que concierne a las leyes estocásticas de los procesos aleatorios y el otro epistemológico, dedicado a estimar grados razonables de creencia en proposiciones carentes de un fondo estadístico" (Hacking, 1975, p. 25).

Revisar el desarrollo histórico de la probabilidad (Coutinho, 2007) da pautas para generar en los escolares un conocimiento gradual a partir de sus errores o dificultades; además permitiría a los profesores tomar conciencia de esos problemas como parte natural de esa evolución conceptual (Batanero, 2005). Por lo tanto en esta investigación se reconocen los significados históricos de la probabilidad señalados por Batanero, Henry y Parzysz (2005): intuitivo, clásico, frecuencial y axiomático.

El enfoque intuitivo de la probabilidad, se ha interpretado como el grado de creencia de una persona en la ocurrencia de los posibles resultados de una situación azarosa (Fischbein, 1975), también refiere al "uso de frases o expresiones coloquiales para cuantificar sucesos inciertos" (Batanero, 2005, p. 253). Toma relevancia la intuición como un programa de acción que es parcialmente autónomo dentro de la cognición y que es una síntesis de la experiencia individual en un dominio dado (Fischbein, 1975). Ya que se vive en un medio ambiente caracterizado por fenómenos estocásticos, desarrollar una intuición de frecuencia es esencial, ésta se genera de modo natural, como resultado de experiencias acumulables del sujeto ante situaciones que implican sucesos aleatorios y que le provocan un conocimiento por la experiencia (Fischbein, 1975).

El enfoque clásico se define como la razón del número de casos favorables respecto al número total de casos posibles, siempre y cuando éstos sean igualmente posibles. Fue instituido por Laplace (1814) y asume la equiprobabilidad de los posibles resultados. Se le puede interpretar como una distribución uniforme de probabilidad.

El enfoque frecuencial se refiere a la estimación de la probabilidad de un evento con base en su frecuencia relativa de ocurrencia en una secuencia grande de repeticiones del fenómeno aleatorio, del cual ese evento es un resultado posible. Es un enfoque a posteriori o empírico (Hawkins y Kapadia, 1984), pues supone la realización de las repeticiones del fenómeno aleatorio, por lo que la probabilidad es el límite hacia el que tiende la frecuencia 
relativa en un número muy grande de repeticiones de ese fenómeno (Fine, 1973).

El enfoque axiomático se estableció como una red de definiciones y relaciones a partir de tres axiomas (Kolmogorov, 1956), una medida de probabilidad $P$ sobre un $\sigma$-campo de subconjuntos $\mathcal{A}$ de un conjunto $\Omega$ es una función con valores reales y dominio en $\mathcal{A}$, tal que: 1) $P(\Omega)=1$;2) $P(A) \geq 0$ para todo $A \in \mathcal{A} ; 3)$ si $A_{n}, n=1,2,3, \ldots$, son conjuntos mutuamente disjuntos en $\mathcal{A}$, entonces:

$$
P\left(\bigcup_{n=1}^{\infty} A_{n}\right)=\sum_{n=1}^{\infty} P\left(A_{n}\right)
$$

Una confluencia de los enfoques frecuencial, clásico y axiomático se muestra por primera vez con el teorema de Bernoulli (Steinbring, 1991) o la ley débil de los grandes números. Ésta consiste en que la probabilidad axiomática se pone en relación con la frecuencial y la clásica, cuando la segunda tiende a la tercera en un número muy grande de ensayos idénticos.

El enfoque intuitivo expresa en menor o mayor grado la probabilidad según la percepción del individuo, por lo que éste podría sentar bases embrionarias para el desarrollo de la probabilidad axiomática (véase la Figura 1), si se tratan a la par los enfoques frecuencial y clásico (Hawkins y Kapadia, 1984). Desarrollar de manera simultánea los tres enfoques, permitiría al individuo establecer elementos para estructurar argumentos matemáticos encaminados a razonar sobre la situación aleatoria que se esté analizando. Es decir, promover la interpretación y comprensión de los procesos y resultados que propuso el estudiante, hacia la evolución de una estructura matemática (Larios, Arellano y González, 2018), desembocarían en estructuras del pensamiento elaboradas en relación a la situación aleatoria de la que se trate.

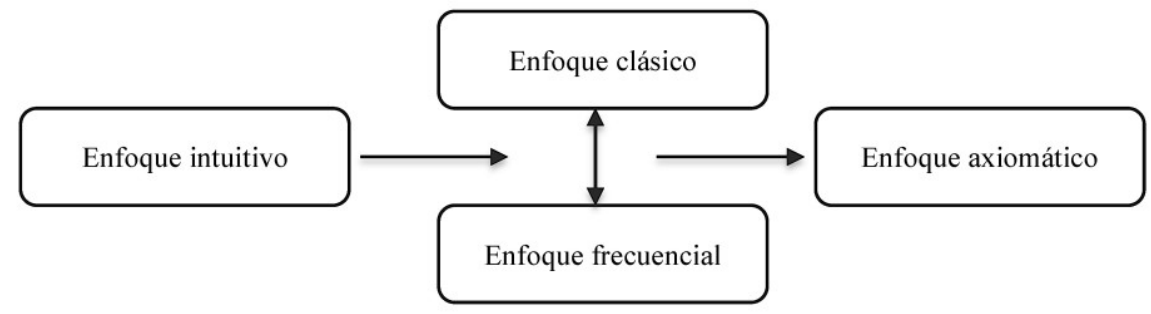

Figura 1: Constitución del enfoque axiomático Fuente: Elaboración propia

Para Sardà (2003) la argumentación refiere a una actividad social, intelectual y verbal que permite justificar una opinión. En tanto Larios, Arellano y González (2018) expresan que un argumento es "el encadenamiento de razonamientos dados por los alumnos para explicar la validez de sus respuestas y procedimientos seguidos para darle solución a un problema" ( $\mathrm{p}$. 
285), en este caso una situación aleatoria. Por lo tanto, interesan los argumentos intuitivos, basados en los grados razonables de creencias según el significado epistemológico de la probabilidad, para explicar la validez de repuestas y cómo se van constituyendo respecto a la experiencia sensorial (Harel y Sowder, 1998) con el fenómeno aleatorio; conciernen así los argumentos intuitivos tendientes a explicar el cómo y por qué de la situación aleatoria, lo que Harel y Sowder (1998) llaman esquema de prueba empírico.

\section{Procedimiento De La Investigación}

De tipo cualitativa (Vasilachis, 2006), la investigación se desarrolló en tres fases (véase la figura 2). En la primera, de indagación, se aplicó un cuestionario diagnóstico para señalar las nociones de probabilidad a una muestra de estudiantes de la licenciatura en enseñanza media con especialidad en matemáticas. En la segunda fase, de enseñanza, se implementaron actividades de probabilidad ex profeso, con miras a ponderar una caracterización matemática del azar (Ojeda, 2003, 2006). En una tercera fase, de comprensión, se aplicó un cuestionario que permitió identificar la comprensión de las ideas fundamentales de probabilidad después de su enseñanza.

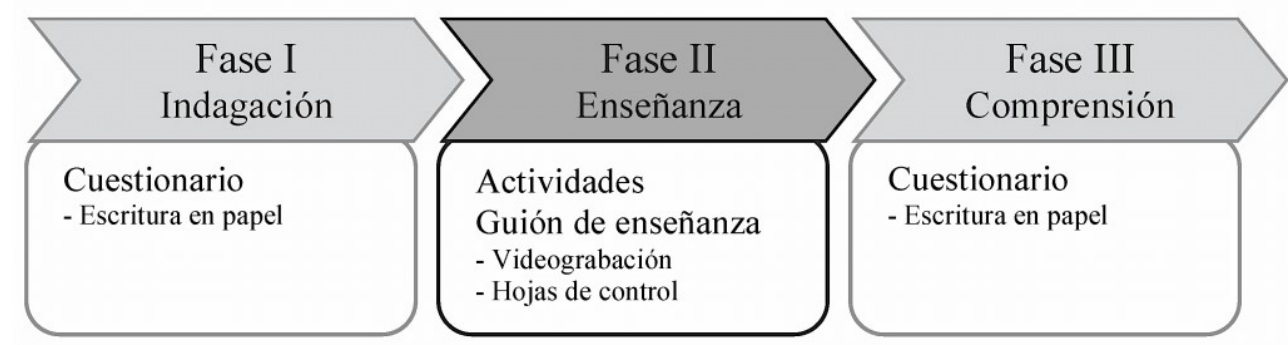

Figura 2: Organización de la investigación en curso Fuente: Elaboración propia

Para este informe de investigación los resultados conciernen a la Fase II de Enseñanza. En la cual se desarrollaron cinco actividades para caracterizar de manera matemática el azar (Ojeda, 2003, 2006), pero para el presente documento sólo se informan los resultados de una. El método fue la experienciación (Maturana, 2003) en estocásticos de los investigadores y los participantes. Los instrumentos fueron las actividades de referencia a fenómenos aleatorios y el guión de enseñanza. Para el registro de los datos se usaron hojas de control y la videograbación, con su respectiva transcripción.

Para tener un mejor acercamiento a la producción de los futuros profesores, se aplicaron criterios de análisis propuestos por Ojeda (2006). Éstos conjugan aspectos de los elementos 
teóricos y de método. Así pues, en el análisis de la presentación de una situación estocástica, para y en la enseñanza, conviene no sólo referirse a los estos conceptos, sino distinguirlos de los otros conceptos matemáticos que se requieren en la situación cuyo foco son los primeros (Ojeda, 2006).

De igual forma, se complementarían los datos provenientes de los recursos semióticos con soporte gráfico (lengua natural escrita, dibujos, gráficas, diagramas, notación matemática) empleados para presentar situaciones con fenómenos aleatorios (Ojeda, 1999), así como el tipo de expresiones que aluden a los estocásticos. Por la naturaleza de la investigación a los criterios se les agregan esquemas de prueba empíricos (Harel y Sowder, 1998) para identificar los argumentos de los futuros profesores. Por lo tanto, para el presente documento, los criterios son:

* Situación de referencia: compete a la relación del individuo con su medio ambiente, el cual condiciona, limita, funda y determina posibilidades (Abbagnano, 1974).

* Ideas fundamentales de estocásticos (Heitele, 1975): permiten tener un análisis conceptual de la situación aleatoria, por lo que son medida de probabilidad, espacio muestra, regla de la adición, regla del producto e independencia, equidistribución y simetría, combinatoria, modelo de urna y simulación, variable estocástica, ley de los grandes números y muestra.

* Argumentos intuitivos: expresiones de aceptación o negación en relación con experiencias sensoriales (esquema de prueba empírico, Harel y Sowder, 1998), relacionados a un conocimiento que se deriva de la experiencia (Fischbein, 1975) aleatoria y estructura un modelo explicativo (Heitele, 1975) en un plano intuitivo.

* Otros conceptos matemáticos: números naturales y el cero, su orden, operaciones aritméticas básicas, razón y proporción, producto cartesiano, por mencionar algunos.

* Recursos semióticos: gráficas, figuras, diagramas, notación matemática, lengua natural escrita. Ojeda (2006) argumenta que se hace referencia a "recursos semióticos" en lugar de "representaciones semióticas", ya que esta última frase corresponde a una interiorización de los usos de esos recursos, que no es inmediata a su presentación (Ojeda, 1999).

* Términos empleados: las palabras y las frases que aluden a estocásticos, ya sea técnicas o cotidianas (Ojeda, 2006, p. 210).

La correlación entre los seis criterios nos permitirá tener una aproximación al análisis de los argumentos que externen en su producción los futuros profesores respecto a la situación 
aleatoria que se desarrolle y a su vez a un acercamiento a su comprensión de la probabilidad.

\subsection{La Situación De Referencia}

Se eligió una actividad que implica el estudio de un fenómeno aleatorio de manera sistemática. Particularmente se pretendió favorecer la idea de azar, así como de las ideas fundamentales de estocásticos como: ley de los grandes números, combinatoria, espacio muestra y medida de probabilidad. La actividad se tomó de la obra de Piaget e Inhelder (1951) acerca del origen de la idea de azar en el niño.

Bajo el enfoque intuitivo, la situación consiste en el balanceo de una bandeja de madera (ver Figura 3) provista de un pivote por debajo, a lo ancho y en el centro de ella; 20 canicas del mismo tamaño de dos colores en igual proporción (diez blancas y diez negras), colocadas a lo ancho en un lado de la bandeja y libres de rodar al lado opuesto en cada balanceo de ésta. Ante el planteamiento del cómo regresarían las canicas y sus posiciones relativas, se espera la producción de los argumentos de los individuos respecto a la situación azarosa.

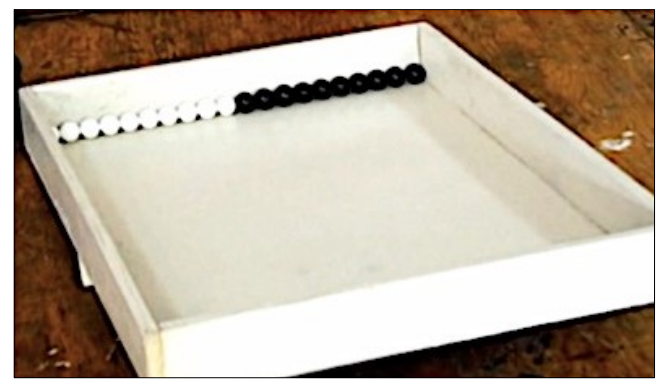

Figura 3: Productor de mezclas aleatorias Fuente: adaptado de Piaget e Inhelder (1951, p. 14)

La actividad promovió la idea de azar, pues por el número relativamente grande de canicas, resulta muy difícil la anticipación de un acomodo particular de ellas al cabo de un balanceo de la bandeja; así como la irreversibilidad de la mezcla, es decir, que además por el número relativamente grande de balanceos es difícil que las canicas regresen a su acomodo inicial. En efecto, el número $(N)$ de maneras en que las 10 canicas indistinguibles, excepto por el color (blancas y negras), se pueden acomodar en los 20 lugares disponibles para ellas es:

$$
N=\frac{n !}{(n-r) ! r !} \times 2=\frac{20 !}{10 ! 10 !} \times 2=369512, r \leq n .
$$

La actividad implica las ideas fundamentales de estocásticos: medida de probabilidad, de manera cualitativa se apela a la idea con la predicción de las posibles posiciones de las canicas como resultado de los balanceos de la bandeja; combinatoria (técnicas de conteo) con 
la petición del dibujo de la anticipación de la posición relativa de las canicas y las trayectorias de las mismas ante cierto número de balanceos; espacio muestra, como el conjunto de las posibles posiciones de las canicas; ley de los grandes números, ya que con la alusión al acomodo resultante de un número muy grande de balanceos y un número relativamente grande de canicas se pretende la identificación de la irreversibilidad de la mezcla; es decir, a un número muy grande de balanceos de la bandeja, el regreso de las canicas a su posición inicial tiene poca probabilidad. La idea de azar resulta de la advertencia de un número muy grande de posiciones relativas (enfoque frecuencial de la probabilidad) de las canicas y de la irreversibilidad de la mezcla (enfoque clásico de la probabilidad).

\subsection{Los Participantes}

La situación de referencia se desarrolló con 12 profesores en formación (20-22 años) del sexto semestre de la Licenciatura en Educación Media especializada en Matemáticas de la Universidad de Colima (UdC). Este programa educativo tiene como objetivo la formación de profesores de matemáticas que incidan en el nivel bachillerato y está enfocado a la educación, pero ofrece cierta especialización en el área de matemáticas. Un acercamiento a la probabilidad la tienen en la unidad de aprendizaje Probabilidad y estadística aplicada a la educación, que entre los propósitos se encuentran: "Explorar las nociones frecuencial y clásica de la probabilidad a través de actividades muy diversas, así como realizar diagramas de árbol para enumerar los posibles resultados de una experiencia aleatoria" (UdC, 2002, p. 180).

Es de notar que los propósitos de la materia sugieren un nivel operativo; es decir, se limitan sólo a la aplicación de reglas de la probabilidad y se descuida la comprensión de ésta. Además, pareciera que, a pesar de mencionar conceptos matemáticos, el enfoque es meramente determinista: realizar diagramas de árbol para enumerar los posibles (UdC, 2002, p. 180; agregamos negritas).

\section{Los Argumentos Intuitivos De Los Futuros Profesores}

La actividad de referencia se aplicó a los participantes en dos sesiones de trabajo con una duración de 30 minutos en promedio. Durante el desarrollo, los futuros profesores dieron evidencia de argumentos hacia la comprensión de las ideas fundamentales de probabilidad: medida de probabilidad, espacio muestra, combinatoria y ley de los grandes números. 
Según la producción, al inicio las expresiones correspondían a la asignación de grados de creencia mediante palabras como "posible", "poco probable", "imposible" (enfoque intuitivo). Conforme se desarrollaba la actividad y, con la interacción constante con el material concreto y un número relativamente grande de ensayos (enfoque frecuencial - enfoque clásico) por parte de ellos, las expresiones aludían a justificaciones del fenómeno aleatorio implicado desde la estructura matemática (enfoque axiomático).

\subsection{Argumentos Sobre Medida De Probabilidad}

Ante la petición de la predicción del acomodo de las canicas después de balancear la bandeja, los futuros profesores hicieron alusión a la medida de probabilidad de manera cualitativa por medio de la asignación de grados de creencia (imposible, poco probable y posible) a los acomodos de las canicas y a las trayectorias de éstas. Por lo que se identificaron tres tipos de argumentos: argumento predictivo imposible, argumento predictivo poco probable y argumento predictivo posible.

El argumento predictivo imposible fue atribuido a la anticipación del acomodo de las canicas por parte de tres futuros profesores, además otros cinco advirtieron que era imposible predecir, anticipar o conocer las trayectorias que tomarían las canicas durante muchos balanceos. Algunas de las expresiones se muestran a continuación, en los episodios de diálogos se muestra la interacción entre el investigador y los estudiantes para profesor diferenciados por la inicial de su nombre.

Investigador: ... ¿Alguien me puede decir que pasó o a qué se debe que el acomodo que ustedes anticiparon de las canicas no es el mismo que se dio después del balanceo?

Alumno J: Yo.

Investigador: ¿Si?

Alumno J: No puedes predecir el movimiento de las canicas porque es algo más bien...

Alumno D: ;Aleatorio! [interrumpe].

Alumno J: Aleatorio, no sé...[pensativo]

Investigador: D, ¿qué pasó? ¿Coincide el acomodo que tú anticipaste con el acomodo que resultó de los cincuenta balanceos?

Alumno D: [Se levanta a observar el contenido de la bandeja] No.

Investigador: ¿A qué se debe?

Alumno D: Pues... yo siento que es algo que no se puede determinar, es imposible, aunque sea un balanceo o sean cincuenta.

Investigador: ¿Por qué? ¿Por qué imposible? A, ¿querías decir algo?

Alumno A: Ah, sí. Yo creo que es imposible identificar con imágenes mentales, quizá haciendo otras cosas...

(Diálogo entre futuros profesores e investigador, 2017).

El argumento predictivo poco probable fue expresado para las mismas acciones que en 
el primer argumento. Los futuros profesores indicaron que era poco probable o no seguro que fueran efectivas las predicciones del acomodo de las canicas emitidas en sus dibujos; otros asignaron la misma medida de probabilidad a realizar la predicción de la trayectoria y acomodo de las canicas. Sobre el regreso de las canicas a su posición original (alineadas y separadas por el color), en mayor medida, tres participantes indicaron que había pocas probabilidades de que sucediera esto.

Respecto al argumento predictivo posible, no se le asigna una probabilidad como tal, pero los futuros profesores consideraron que esto podía suceder en algún momento o de alguna manera. En sus respuesta, seis indicaron que era posible o probable que las canicas regresaran a su posición original. De estos futuros profesores, algunos entraron en discusión sobre dicho suceso, en sus argumentos iniciaban en considerar el número de balanceos necesarios para obtener la posición inicial (canicas blancas y canicas negras agrupadas por color).

Investigador: ... Si realizamos muchos, muchos otros balanceos, ¿pueden regresar a la posición inicial donde estaban estas canicas?

Alumno A: Pero no creo que regresen a su posición inicial.

Alumno V: Yo digo que sí se puede mientras no haya tantas canicas.

Alumno L: Y mientras no modifiques el tablero este... Y puedas hacer tantos balanceos hasta que por fin te dé el inicial. [Murmullos].

Alumno D: No, es que estamos alegando que es un ciclo...

Alumno A: Es un ciclo.

Alumno D: Pero lo que yo digo es que a lo mejor sí pueden llegar otra vez las diez blancas y las diez negras, va a ser no sé, en " $n$ " eventos, pero el problema es que no es un ciclo porque si das otro más tendría que repetirse el primer balanceo que la primera posición. Pero sí se puede, a lo mejor a los setenta puedes llegar a las diez blancas y a las diez negras...

Alumno A: O setenta y ocho.

Alumno D: Y vuelves a empezar, ¿no? Otra vez a los balanceos y ahora a los ciento veinte balanceos otra vez diez y diez [refiere al acomodo inicial de las canicas], entonces no es algo que se pueda terminar.

(Diálogo entre futuros profesores e investigador, 2017).

Como se podrá identificar, la evolución de los argumentos de los estudiantes para profesor, ante la necesidad de explicar la situación aleatoria, evocan a otras justificaciones como los choques entre las canicas o con las paredes de la bandeja, la fuerza que se imprime en el movimiento, el viento, etc., y no sólo refieren a la intervención del azar como única respuesta.

Alumno D: Porque hay muchos elementos o variantes de... que afectan ese evento. Por ejemplo, el viento que le dé a una canica y ya se mueve para un lado o...

Alumno G: Que vaya lento o que vaya rápido.

Alumno D: ¡Aha! La fuerza que imprimas en cada uno de los balanceos no es la misma que imprimes en todos.

(Diálogo entre futuros profesores e investigador, 2017). 


\subsection{Argumentos Sobre Espacio Muestra}

Los tipos de argumentos para esta idea fundamental, están relacionados a la predicción de las posiciones relativas de las canicas en conjunto, al cabo de cierto número de balanceos (cinco balanceos, cincuenta balanceos y muchos balanceos). El investigador promovió la reflexión sobre el dibujo realizado por los futuros profesores, pues éste corresponde a una posición del conjunto de muchas posiciones posible que podrían tomar las canicas.

Se solicitó la predicción del conjunto de canicas después de realizar un balanceo. Los participantes anticiparon mediante un dibujo esas posiciones relativas de manera lineal (cuatro futuros profesores); es decir, todas formando una fila revueltas entre ellas o con el mismo orden y sugirieron que su trayectoria sería rectilínea. De los doce futuros profesores, solo cinco anticiparon un acomodo distinto a la inicial, advirtieron de los choques entre las canias y las caras laterales de la bandeja. Por lo anterior, los tipos de argumentos que se identificaron fueron: argumento predictivo de posición lineal y argumento predictivo de posición no lineal.

Después de la primera interacción con la bandeja (primer balanceo), en las siguientes predicciones los participantes anticiparon acomodos lineales y no lineales; es decir, las canicas ordenadas en una sola línea o unas sobre otras, respectivamente. También identificaron la mezcla de las canicas e indicaron que en la trayectoria de las canicas, durante los balanceos, chocaban entre ellas y con los bordes de la bandeja; del mismo modo, atribuían los acomodos y trayectorias de las canicas a fuerzas externas como el viento, la fuerza impresa en los balanceos, la gravedad, lo que sugiere una evolución al tipo de justificaciones; algunas de las producciones se pueden observar en la Figura 4.
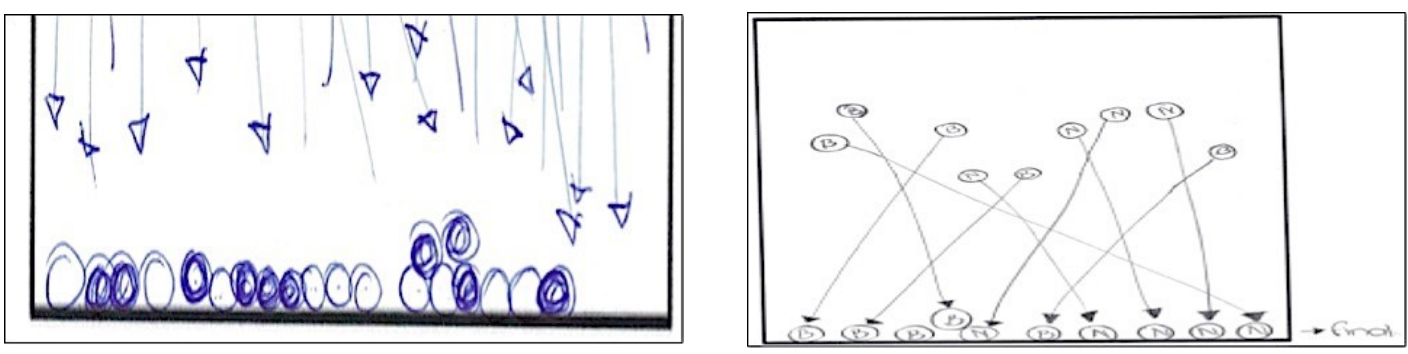

Figura 4: Tipos de producciones según los argumentos lineal y no lineal Fuente: datos del estudio

\subsection{Argumentos Sobre Combinatoria}

Los tipos de argumentos que emitieron los futuros profesores respecto a la combinatoria, corresponden a acomodos lineales (cinco participantes), en un primer momento, y posterior aun 
balanceo, sus acomodos incluyeron amontonamiento de canicas (tres participantes). También se reveló cierto patrón de orden de las posiciones y aseguraban que entre más balanceos, más revueltas quedaban las canicas (cuatro participantes); es decir identificaron la mezcla.

Hubo predicciones de acomodos de canicas que de ninguna manera resultarían pues no mantenían la cantidad de canicas utilizadas en la bandeja o aunque mantenían la cantidad total de canicas, el número de canicas por color no correspondía al empleado en la bandeja. Por lo que los tipos de argumentos que se identificaron fueron: argumento de acomodo lineal, argumento de acomodo con patrón, argumento de acomodo conglomerado y argumento de acomodo azaroso.

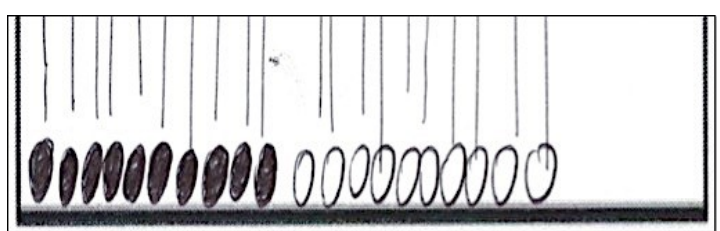

a) Acomodo lineal

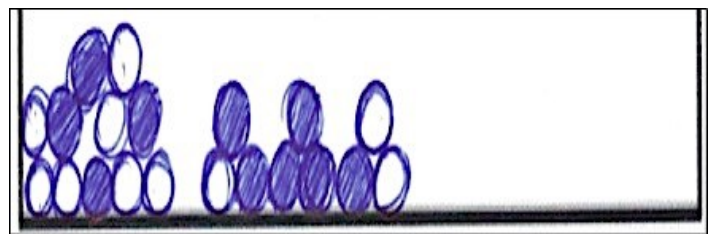

c) Conglomerado

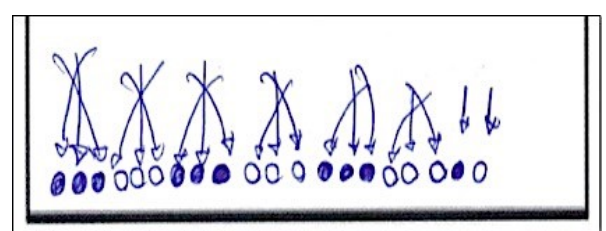

b) Acomodo con patron

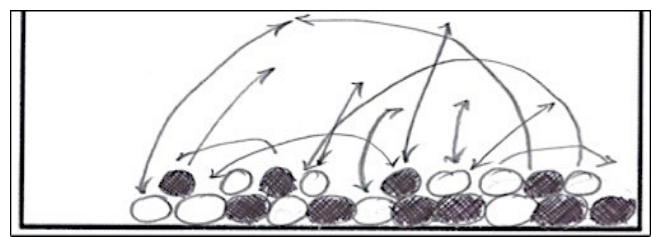

d) Acomodo azaroso

Figura 5: Tipos de acomodos anticipados por los participantes Fuente: datos del estudio

Con la petición de la predicción del acomodo de las canicas después de cincuenta balanceos por medio de un dibujo, los participantes indicaron predicciones azarosas respecto las primeras producciones. Esto es, la experiencia de la interacción con la bandeja influyó directamente en los futuros profesores para ampliar sus argumentos respecto al contenido matemático (ver Figura 5).

\subsection{Argumentos Sobre La Ley De Los Grandes Números}

Los futuros profesores evidenciaron un acercamiento a la irreversibilidad de la mezcla. Se preguntó si era posible que las canicas regresaran a la posición inicial cuando la bandeja se balanceara muchas, muchas veces, a lo que respondieron que era casi imposible o muy poco probable. Por lo que, se tienen argumento de reversibilidad y argumento de irreversibilidad. Sus argumentos evolucionaron a justificaciones con estructura matemática, por ejemplo el reconocer que una probabilidad pequeña puede ocurrir siempre y cuando exista.

Investigador: Ok, gracias. Chicos, entonces si nosotros balanceamos muchas, muchas, muchas 
veces más este... la bandeja, ¿ustedes consideran que las canicas regresarian a su posición inicial, es decir, diez blancas y diez negras?

Todos: Nooo...

Alumno L: Sí, chance sí, pero es...

Alumno D: Espera...

Alumno L: Es que yo... Algo asi puse en mi predicción, pero no exactamente. Quedaban negras, digo, nueve negras y quedaba una blanca y una negra y después las otras nueve [refiere a las blancas]. Yo tenía esa predicción, pero no sé, creo que sí se podría, pero sería una probabilidad muy muy muy pequeña de que quedaran así.

Alumno K: Mmm ...[Mueve la cabeza en tono de desacuerdo].

Alumno M: Bueno, pues mientras exista la probabilidad si puede pasar, pero es poca, es pequeña.

(Diálogo entre futuros profesores, 2017).

Un resumen de los tipos de argumentos intuitivos que produjeron los futuros profesores se presenta en la Tabla 1. Respecto al número de participantes, estos varían en relación a las anticipaciones de los movimientos (uno, cinco, cien) de la bandeja.

Tabla 1: Caracterización de los argumentos intuitivos por idea fundamental

\begin{tabular}{cl}
\hline $\begin{array}{c}\text { Ideas fundamentales de } \\
\text { estocásticos }\end{array}$ & \multicolumn{1}{c}{ Tipos de argumentos intuitivos } \\
\hline \multirow{2}{*}{ Medida de probabilidad } & $\begin{array}{l}\text { Argumento predictivo imposible } \\
\text { Argumento predictivo poco probable } \\
\text { Argumento predictivo posible }\end{array}$ \\
& $\begin{array}{l}\text { Argumento predictivo de posición lineal } \\
\text { Espacio Muestra }\end{array}$ \\
& $\begin{array}{l}\text { Argumento predictivo de posición no lineal } \\
\text { Argumento de acomodo lineal }\end{array}$ \\
& Argumento de acomodo con patrón \\
Combinatoria & Argumento de acomodo azaroso \\
& Argumento de reversibilidad \\
& Argumento de irreversibilidad \\
\hline
\end{tabular}

Fuente: Elaborado por los autores

\section{A Manera De Conclusión}

Después de la aplicación de la actividad mezcla aleatoria, los elementos teóricos permitieron identificar los argumentos intuitivos respecto a la probabilidad, particularmente medida de probabilidad, espacio muestra, combinatoria y ley de los grandes números. Se mostró una evolución de justificaciones intuitivas causales a justificaciones matemáticas. En correspondencia a lo que determinan Piaget e Inhelder (1951), los futuros profesores transitaron en los tres estadios en las sesiones de trabajo: de ver el conjunto de canicas como un solo objeto a señalar las posiciones relativas de éstas, sus trayectorias aleatorias y la irreversibilidad. 


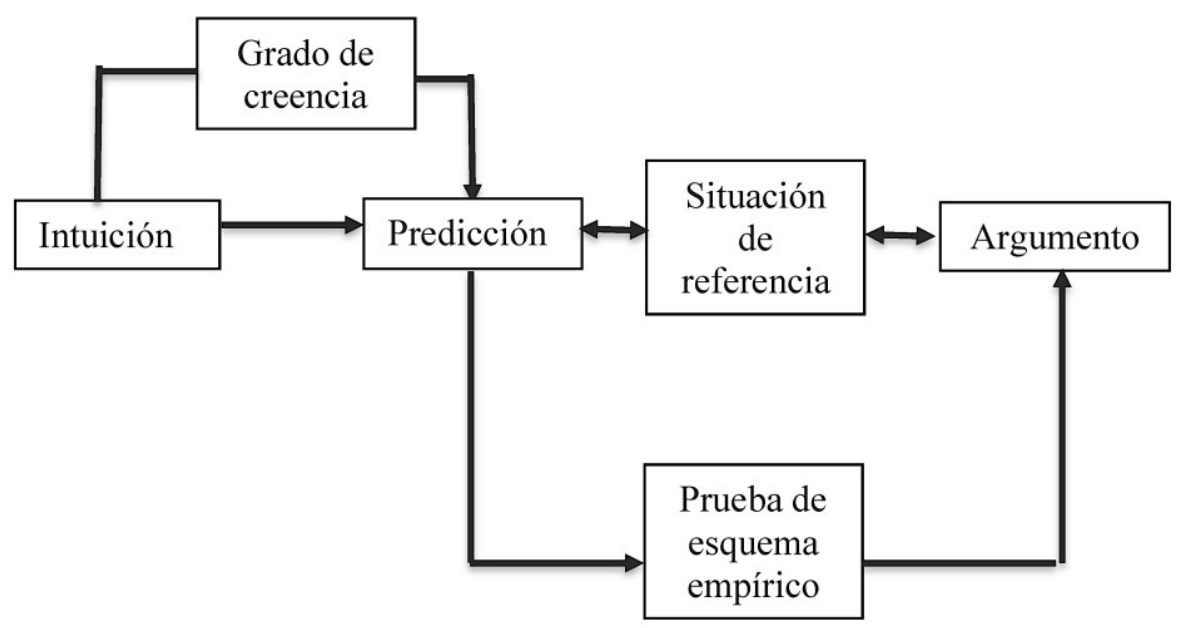

Figura 6: Esquema de argumento empírico para la mezcla aleatoria Fuente: adaptado de Harel y Sowder (1998)

La evolución de los argumentos estuvo caracterizada por la intuición de frecuencia (Fischbein, 1975) al realizar los ensayos con el material concreto y que, ante la predicción, provocó el surgimiento de grados de creencia (Fischbein, 1975; Batanero, 2005). Lo anterior desembocó en pruebas de esquema empírico (Harel y Sowder, 1998) dada la experiencia con el fenómeno aleatorio, por lo que se configuró el argumento (Larios, Arellano y González, 2018; Sardà, 2003) intuitivo. En el esquema de la Figura 6, se presenta la evolución lógica de las expresiones emitidas por los futuros profesores para consolidar sus argumentos.

Respecto a medida de probabilidad, los argumentos intuitivos presentan un acercamiento a la comprensión tanto de manera cualitativa como cuantitativa, pues asignaron grados de creencia por medio de expresiones como "posible", "poco probable" e "imposible" (Fischbein, 1975; Batanero, 2005), las asignaciones numéricas que declaraban como probabilidad poniendo en relación la cardinalidad del evento con la cardinalidad del espacio muestra, de esa manera normaban la probabilidad (Heitele, 1975) como una función en el intervalo $[0,1]$.

En la actividad de enseñanza interesó el balanceo de la bandeja con las 20 canicas (10 blancas y 10 negras) y todas las posiciones relativas de éstas; además de la predicción por medio de dibujos, se apeló a las ideas de combinatoria, ley de los grandes números, espacio muestra y medida de probabilidad. El recurso semiótico permitió recuperar elementos de la situación aleatoria que se escapaban a la simple vista y sometió al análisis el fenómeno aleatorio implícito (Ojeda, 1999).

Según Fischbein (1975) la intuición de frecuencia se desarrolla naturalmente, con experiencias individuales acumulables de situaciones aleatorias. En ese caso, la actividad de 
enseñanza ofreció a los futuros profesores el acercamiento a un aprendizaje probabilístico con la comparación de sus producciones anticipadas (enfoque clásico) con lo que resultó efectivamente al cabo de los ensayos (enfoque frecuencial). Por lo tanto, se favoreció un conocimiento promovido por la experiencia y permitió abonar a los argumentos de los individuos para formalizar un conocimiento matemático (enfoque axiomático).

Steinbring (1989) argumenta una necesidad de tratar los conceptos de estocásticos en una estrecha relación con marcos de referencia que permitan acentuar la naturaleza de la aleatoriedad y de la incertidumbre. Para la referencia al objeto se utilizó material físico, pues Steinbring (2005) considera importante diferenciar la situación que implica al objeto, de los signos o símbolos empleados en la presentación de la actividad y en su desarrollo, para poner en juego el concepto matemático. Como lo señaló Fischbein (1975), la exposición a situaciones concretas que revisten una acumulación de experiencias provocan en el individuo, de manera natural, la intuición de frecuencia relativa.

\section{Agradecimientos}

El presente informe de investigación se realizó en el marco del proyecto SEP-PRODEP DSA/103.5/15/6897.

\section{Referencias}

Abbagnano, N. (1974). Diccionario de filosofía (1 $1^{\mathrm{a}}$ ed). México: Fondo de cultura económica.

Batanero, C. (2005). Significados de la probabilidad en la educación secundaria. Revista Latinoamericana de Matemática Educativa, 8(3), 247-263.

Batanero, C. y Borovcnik, M. (2016). Statistics and Probability in High School. Rotterdam: Sense Publishers

Batanero, C., Henry, M. y Parzysz, B. (2005). The nature of chance and probability. In G.A. Jones (Ed.), Exploring probability in school: Challenges for teaching and learning (1642). New York: Springer.

Coutinho, C. (2007). Conceitos probabilísticos: quais contextos a história nos aponta? REVEMAT: Revista Eletrônica de Educação Matemática, 2(1), 50-67. Doi: https://doi.org/10.5007/\%25x

Fine, T. (1973). Theories of probability: an examination of foundations. Londres: Academic Press.

Fischbein, E. (1975). The intuitive sources of probabilistic thinking in children. Holland: Reidel. 
Hacking, I. (1975). The emergence of probability. USA: Cambridge University Press.

Harel, G. y Sowder, L. (1998). Students' proof schemes: Results from exploratory studies. En A. Schoenfeld, J. Kaput y E. Dubinsky (Eds.) Research in collegiate mathematics education III (234-282). USA: AMS.

Hawkins, A. y Kapadia, R. (1984). Children's conceptions of probability a psychological and pedagogical review. Educational Studies in Mathematics, 15(1), 349-377. Doi: https://doi.org/10.1007/BF00311112

Heitele, D. (1975). An epistemological view on fundamental stochastic ideas. Educational Studies in Mathematics, 6(2), 187-205. Doi: https://doi.org/10.1007/BF00302543

Holmes, P. (1980). Teaching Statistics 11-16. UK: Foulsham Educational.

Kahneman, D., Slovic, P. y Tversky, A. (1982). Judgment under uncertainty: Heuristics and biases. New York: Cambridge University Press.

Kolmogorov, A. (1956). Fundations of the theory of probability. New York: Chelsea Publishing.

Laplace de, P. S. (1812; 1820). Théorie analytique des probabilités ( $3^{\text {a }}$ edición). Francia: Courcier.

Larios, V., Arellano, C. y González, N. (2018). Análisis de argumentos producidos por alumnos de bachillerato al resolver problemas de geometría. REDIMAT - Journal of Research in Mathematics Education, 7(3), 280-310. Doi: http://dx.doi.org/10.17583/redimat.2018.2343

López-Mojica, J. M., Ojeda, A. M. y Salcedo, J. (2018). Ideas fundamentales de estocásticos en libros de texto de educación primaria: una alternativa de enseñanza. IE Revista de Investigación Educativa de la REDIECH, 9(17), 87-102.

Martínez, A. M. y Ojeda, A. M. (2017). Comprensión de la media ponderada por docentes en formación para primaria. En Serna, L. (Ed.). Acta Latinoamericana de Matemática Educativa Vol. 30 (1125-1136). México: Comité Latinoamericano de Matemática Educativa.

Maturana, H. (2003). Desde la Biología a la Psicología. Argentina: Lumen.

Narens, L. (2007). Theories of probability an examination of logical and qualitative foundations. USA: World Scientific.

Ojeda, A. M. (1994). Understanding fundamental ideas of probability at pre-university levels. (Tesis Doctoral). King's College London. UK.

Ojeda, A. M. (1999). Concept and representation in the research on probability education. In F. Hitt, M. Santos (Ed.), Proceedings of the Psychology of Mathematics Education, North American Chapter XXI (83-96). México: ERIC.

Ojeda, A. M. (2003). Azar y grandes números en didáctica de la probabilidad. En E. Filloy (Ed.) Matemática Educativa. Aspectos de la Investigación Actual (158-173). México: CinvestavFondo de Cultura Económica. 
Ojeda, A. M. (2006). Estrategia para un perfil nuevo de docencia: un ensayo en la enseñanza de estocásticos. En E. Filloy (Ed.) Matemática Educativa, treinta años (195-214). México: Santillana-Cinvestav.

Piaget, J. (1982). Le possible et le nécéssaire. Francia: PUF.

Piaget, J. e Inhelder, B. (1951). La Génèse de l'idée de Hasard Chez l'enfant. París: PUF.

Sardá, A. (2003). Argumentar: Proposar i validar models. En N. Sanmartí (coord.), Aprendre Ciencies tot aprenent a escriure ciencia (149- 168). Barcelona: Ediciones 62.

Steinbring, H. (1989). The interaction between teaching practice and theoretical conceptions. A cooperative model of in-service training in stochastics for mathematics teachers (Grades 510). En R. Morris (Ed.) Studies in Mathematics Education. The Teaching of Statistics (202214). París: UNESCO.

Steinbring, H. (1991). The concept of chance in everyday teaching: aspects of a social epistemology of mathematical knowledge. Educational Studies in Mathematics, 22(1), 503-522. Doi: https://doi.org/10.1007/BF00312713

Steinbring, H. (2005). The construction of new mathematical knowledge in classroom interaction. USA: Springer.

Universidad de Colima (2002). Licenciatura en Educación Media Especializada en Matemáticas. México: UdC.

Vasilachis, I. (2006). Estrategias de investigación cualitativa. Barcelona: Gedisa.

Vásquez, C. y Alsina, A. (2017). Lenguaje probabilístico: un camino para el desarrollo de la alfabetización probabilística. Bolema: Boletim de Educação Matemática, 31(57), 454 478. Doi: http://dx.doi.org/10.1590/1980-4415v31n57a22 